\title{
Diagnostic Value of the Serum Galactomannan and $(1,3)-\beta$-D-glucan Assays for Invasive Pulmonary Aspergillosis in Non-neutropenic Patients
}

\author{
Xuejiu Cai ${ }^{1,2}$, Wentao $\mathrm{Ni}^{1}$, Chuanqi Wei ${ }^{1}$ and Junchang Cui ${ }^{1}$
}

\begin{abstract}
Objective Galactomannan (GM) and (1,3)- $\beta$-D-glucan (BG) are considered useful seromarkers for the diagnosis of invasive pulmonary aspergillosis (IPA) in patients with neutropenia. However, there is still limited data on these seromarkers for testing non-neutropenic patients who are at the risk of IPA. The aim of this study was to evaluate the value of these two serum antigen assays for the early diagnosis of IPA in patients without neutropenia.

Methods Between January 2011 and December 2012, 97 patients with suspected IPA admitted to the department of respiratory diseases and the respiratory intensive care unit were prospectively monitored. Serum GM and BG assays were performed before the patients received antifungal therapy.

Results Patients were classified as proven IPA $(n=11)$, probable IPA $(n=16)$, possible IPA $(n=4)$, or nonIPA $(n=66)$. The most common underlying disease of patients with IPA was chronic obstructive pulmonary disease $(18.5 \%)$, and $22.2 \%$ patients with IPA had no known diseases. The sensitivities, specificities, and positive and negative predictive values of the GM and BG assays and at least one positive on both assays were $40.7 \% / 89.4 \% / 61.1 \% / 78.7 \%, 48.1 \% / 78.8 \% / 48.1 \% / 78.8 \%$, and $70.4 \% / 75.8 \% / 54.3 \% / 86.2 \%$, respectively.

Conclusion Compared with the testing of neutropenic patients, the serum GM or BG assay alone was less useful for the diagnosis of IPA in non-neutropenic patients. However, at least one positive result of the two serum assays appeared to be useful in the diagnosis of IPA in non-neutropenic patients.
\end{abstract}

Key words: galactomannan, $(1,3)-\beta$-D-glucan, invasive pulmonary aspergillosis, non-neutropenic patients

(Intern Med 53: 2433-2437, 2014)

(DOI: 10.2169/internalmedicine.53.2381)

\section{Introduction}

Invasive pulmonary aspergillosis (IPA) is most common in patients with hematological diseases, particularly in those with prolonged neutropenia (1). However, an increasing number of studies suggests that IPA is not rare in nonneutropenic patients and can cause high morbidity and mortality (2-4). Early diagnosis is the key to improve prognosis. However, the clinical features of IPA are not well defined in non-neutropenic patients, especially in those without underlying conditions (5). Sputum cultures may require several days of growth, and thoracic computed tomography (CT) scanning is not sensitive to the halo and air crescent signs (6). The invasive procedures required to obtain specimens for histopathological examination can only be performed in a subset of patients. Thus, accurate and early diagnosis to initiate prompt antifungal therapy still remains a great challenge for physicians.

The value of serum galactomannan (GM) and $(1,3)-\beta-D-$ glucan (BG) assays are well documented for the early diagnosis of IPA in patients with neutropenia (7-9). However, there is limited data on these serum markers for testing nonneutropenic patients who are at risk of IPA, and the value of the combination of both tests for this patient population remains unclear. In the present study, we evaluated the useful-

${ }^{1}$ Department of Respiratory Diseases, Chinese People's Liberation Army General Hospital, China and ${ }^{2}$ Department of Respiratory Diseases, Guangzhou General Hospital of Guangzhou Army Command of Chinese People's Liberation Army, China

Received for publication December 24, 2013; Accepted for publication April 6, 2014

Correspondence to Dr. Junchang Cui, guoguoyoumeng@163.com 
Table 1. Clinical Characteristics of 97 Patients who Underwent Galactomannan and $(1,3)-\beta$-D-Glucan Assays for the Diagnosis of IPA

\begin{tabular}{lccc}
\hline & IPA & Study cases & p value \\
& $(\mathrm{n}=27), \mathrm{n}(\%)$ & $(\mathrm{n}=97), \mathrm{n}(\%)$ & 0.201 \\
Age, y, median (range) & $60(31-79)$ & $56(21-84)$ & 0.712 \\
Male & $15(55.6)$ & $60(61.9)$ & \\
Underlying disease & & & \\
Solid organ malignancy & $3(11.1)$ & $7(7.2)$ & 0.797 \\
Kidney transplant & $1(3.7)$ & $10(10.3)$ & 0.493 \\
Rheumatologic disease & $2(7.4)$ & $9(9.3)$ & 0.936 \\
Kidney non-malignant disease & $1(3.7)$ & $5(5.2)$ & 0.844 \\
Diabetes mellitus & $3(11.1)$ & $7(7.2)$ & 0.797 \\
Old pulmonary tuberculosis & 0 & $3(3.1)$ & 1.000 \\
Cardiovascular disease & $1(3.7)$ & $9(9.3)$ & 0.588 \\
Non-malignant pulmonary disease & $10(37.0)$ & $29(29.9)$ & 0.637 \\
Without basic diseases & $6(22.2)$ & $18(18.6)$ & 0.880 \\
Immunosuppressive therapy & $3(11.1)$ & $19(19.6)$ & 0.462 \\
Long-term steroid treatment $(\geq 3$ weeks) & $12(44.4)$ & $32(33.0)$ & 0.383 \\
\hline IPA: invasive pulmonary aspergillosis & \multicolumn{3}{l}{}
\end{tabular}

ness of GM and BG assays for the early diagnosis of IPA in non-neutropenic patients and investigated whether the combination of both tests would improve the diagnostic efficiency.

\section{Materials and Methods}

\section{Study design}

The study was conducted between January 2011 and December 2012 in the department of respiratory diseases and the respiratory intensive care unit of Chinese PLA General Hospital in Beijing, China. Patients (>18 years old) with newly emerging respiratory symptoms or those in the acute exacerbation stage with lung shadows on CT (e.g., infiltrative shadow, ground-glass opacity, multiple nodules, the halo and air crescent signs, etc.) and without improved clinical symptoms or imaging findings after four days of broadspectrum antibiotic treatment were included in the study. The exclusion criteria were chronic necrotizing pulmonary aspergillosis (CNPA), aspergilloma, neutropenia, and hematological diseases. CNPA and aspergilloma were differentiated from IPA mainly according to the clinical and radiological findings, such as patients with a slowly progressive course of weeks to months with chest CT showing pleural thickening and cavitary lesions, particularly in the upper lung lobes. Each of the included patients was classified according to the criteria of the European Organization for Research and Treatment of Cancer/Mycoses Study Group (EORTC/MSG) revised in 2008 (1). The patients were categorized as proven, probable, possible, or non-IPA. Only proven and probable IPA were considered truly positive, and only no-IPA cases were considered truly negative. The definite diagnosis of IPA was not based on the results of the GM or BG test.

\section{1-3- $\beta$-D-Glucan and galactomannan detection}

Serum samples of all the included patients were collected before they received any antifungal therapy. Serum samples were simultaneously tested with the BG and GM assays. BG was detected with the Glucatell test kit (Associates of Cape Cod, Falmouth, USA). The patients were considered positive if the index value was $\geq 80 \mathrm{pg} / \mathrm{mL}$ (10). The detection of the GM antigen was performed with the Platelia Aspergillus EIA test (Bio-Rad Laboratories, Hercules, USA) according to the manufacturer's instructions (11). A sample was judged positive if the level of GM was $\geq 0.5$. GM and $\mathrm{BG}$ antigen testing was performed in batches.

\section{Date analysis}

The sensitivity, specificity, positive predictive value (PPV), negative predictive value (NPV), and accuracy of GM and BG assays were calculated in comparison with the reference diagnosis, which was based on cases of proven or probable IPA. Student's t-tests or the Wilcox rank-sum test were used to compare continuous variables, and Chi-square or Fisher's exact tests were used to compare categorical variables. All $\mathrm{p}$ values were 2 -tailed, and $\mathrm{p}<0.05$ was considered statistically significant.

\section{Results}

\section{Study population}

During the study period, 97 patients were included. Patients were classified as proven IPA $(n=11)$, probable IPA $(n=16)$, possible IPA $(n=4)$, or non-IPA $(n=66)$. Their clinical characteristics are shown in Table 1. The median age was 56 years (range $21-84$ years), and $61.9 \%$ of the patients were men. The most common underlying diseases were nonmalignant pulmonary disease $(29.9 \%)$, followed by no underlying disease (18.6\%), and kidney transplant (10.3\%).

Table 2 shows the characteristics of 27 patients with proven $(n=11)$ or probable $(n=16)$ IPA. Six patients had no underlying diseases. Non-malignant pulmonary disease (10/ 27) was the most common underlying condition of patients with IPA. Among these patients, five had chronic obstructive pulmonary disease (COPD), two had bronchiectasis, two had asthma, and one had interstitial pulmonary fibrosis. In addition, three patients had solid organ malignancy, and three had diabetes mellitus. Three patients had received immunosuppressive therapy for related diseases, and 12 patients had inhaled and/or systemically used corticosteroids for more than three weeks prior to the diagnosis of IPA. The mortality rate of the 27 patients with proven or probable IPA was $33.3 \%$.

\section{Diagnostic and prognostic values of the serum GM and $B G$ assays}

The results of the serum GM and BG tests are shown in Tables 3 and 4. The GM and BG assays of the study population ( $\mathrm{n}=93$, four patients with possible IPA were excluded) yielded positive values in 18 and 27 patients, respectively. At least one of the assays was positive for 35 patients, and 
Table 2. Clinical Characteristics of 27 Patients with Proven or Probable IPA

\begin{tabular}{|c|c|c|c|c|c|c|c|c|c|c|}
\hline No. & Sex & $\begin{array}{l}\text { Age } \\
\text { (y) }\end{array}$ & $\begin{array}{l}\text { Underlying } \\
\text { Condition }\end{array}$ & $\begin{array}{l}\text { EORTC/MSG } \\
\text { for IPA }\end{array}$ & $\begin{array}{l}\text { Risk factor } \\
\text { for IPA }\end{array}$ & Specimen $^{*}$ & $\begin{array}{l}\text { WBC } \\
\left(10^{3} / \mathrm{mL}\right)\end{array}$ & $\begin{array}{l}\mathrm{BG} \\
(\mathrm{pg} / \mathrm{mL})\end{array}$ & GM & Death \\
\hline 1 & M & 46 & Bronchiectasis & Proven & - & Tissue & 5.21 & 260.3 & 0.20 & No \\
\hline 2 & M & 71 & Lung cancer & Proven & - & Tissue & 5.63 & 23.1 & 0.42 & Yes \\
\hline 3 & M & 55 & COPD & Proven & Steroid & Tissue & 7.74 & 15.9 & 0.25 & No \\
\hline 4 & M & 60 & COPD & Proven & Steroid & Tissue & 9.97 & 976.3 & 0.21 & Yes \\
\hline 5 & M & 66 & $\mathrm{DM}$ & Proven & - & Tissue & 5.05 & 50.5 & 0.39 & No \\
\hline 6 & $\mathrm{~F}$ & 55 & - & Proven & - & Tissue & 6.49 & 88.4 & 0.47 & No \\
\hline 7 & M & 59 & - & Proven & - & Tissue & 10.75 & 335.8 & 0.27 & No \\
\hline 8 & M & 56 & Kidney cancer & Proven & - & Tissue & 6.81 & 175.2 & 0.40 & Yes \\
\hline 9 & M & 39 & - & Proven & Steroid & Tissue & 5.73 & 10.0 & 3.29 & No \\
\hline 10 & $\mathrm{~F}$ & 58 & Hypertension & Proven & - & Tissue & 4.42 & 35.5 & 0.77 & No \\
\hline 11 & M & 43 & - & Proven & - & Tissue & 5.47 & 25.4 & 0.18 & No \\
\hline 12 & $\mathrm{~F}$ & 76 & Asthma & Probable & Steroid & BAL & 7.67 & 81.4 & 0.25 & Yes \\
\hline 13 & $\mathrm{~F}$ & 74 & Colon cancer & Probable & - & BAL & 13.81 & 42.6 & 1.33 & Yes \\
\hline 14 & $\mathrm{~F}$ & 56 & Bronchiectasis & Probable & - & BAL & 7.45 & 125.6 & 0.88 & No \\
\hline 15 & $\mathrm{~F}$ & 31 & - & Probable & - & BAL & 6.99 & 657.1 & 0.42 & No \\
\hline 16 & M & 78 & COPD & Probable & Steroid & BAL & 4.23 & 16.6 & 1.05 & No \\
\hline 17 & M & 67 & Nephrotic syndrome & Probable & Steroid & Sputum & 7.47 & 15.8 & 0.46 & No \\
\hline 18 & M & 79 & COPD & Probable & Steroid & Sputum & 7.52 & 56.5 & 1.82 & Yes \\
\hline 19 & $\mathrm{~F}$ & 71 & COPD & Probable & Steroid & Sputum & 10.42 & 28.6 & 0.34 & No \\
\hline 20 & M & 36 & Kidney TPL & Probable & Steroid IST & Sputum & 16.86 & 185.5 & 0.57 & No \\
\hline 21 & $\mathrm{~F}$ & 67 & $\mathrm{DM}$ & Probable & Steroid & Sputum & 8.55 & 895.2 & 1.62 & Yes \\
\hline 22 & M & 62 & $\mathrm{DM}$ & Probable & - & Sputum & 7.89 & 407.2 & 0.54 & No \\
\hline 23 & $\mathrm{~F}$ & 66 & RA & Probable & - & Sputum & 11.58 & 283.5 & 0.25 & No \\
\hline 24 & $\mathrm{~F}$ & 75 & IPF & Probable & Steroid IST & Sputum & 6.24 & 25.2 & 0.92 & Yes \\
\hline 25 & $\mathrm{~F}$ & 52 & - & Probable & - & Sputum & 18.39 & 75.2 & 0.26 & No \\
\hline 26 & $\mathrm{~F}$ & 75 & Asthma & Probable & - & Sputum & 9.09 & 68.4 & 0.33 & No \\
\hline 27 & M & 46 & Dermatomyositis & Probable & Steroid IST & Sputum & 8.06 & 72.5 & 1.13 & Yes \\
\hline
\end{tabular}

*The sample from where the Aspergillus species was isolated.

EORTC/MSG: European Organization for Research and Treatment of Cancer/Mycoses Study Group, IPA: invasive pulmonary aspergillosis, BG: (1, 3)- $\beta$-D-glucan, GM: galactomannan, COPD: chronic obstructive pulmonary disease, DM: diabetes mellitus, TPL: transplantation, RA: rheumatoid arthritis, IPF: interstitial pulmonary fibrosis, IST: immunosuppressive therapy, BAL: bronchoalveolar lavage

Table 3. Performance of GM Assay, BG Assay and the Combination in Detection of IPA

\begin{tabular}{lcccc}
\hline & Group & & \\
\cline { 2 - 5 } & BG & GM & BG/GM & BG+GM \\
\hline Sensitivity (\%) & $48.1(13 / 27)$ & $40.7(11 / 27)$ & $70.4(19 / 27)$ & $18.5(5 / 27)$ \\
Specificity (\%) & $78.8(52 / 66)$ & $89.4(59 / 66)$ & $75.8(50 / 66)$ & $92.4(61 / 66)$ \\
PPV (\%) & $48.1(13 / 27)$ & $61.1(11 / 18)$ & $54.3(19 / 35)$ & $50.0(5 / 10)$ \\
NPV (\%) & $78.8(52 / 66)$ & $78.7(59 / 75)$ & $86.2(50 / 58)$ & $73.5(61 / 83)$ \\
Accuracy (\%) & $69.9(65 / 93)$ & $75.3(70 / 93)$ & $74.2(69 / 93)$ & $71.0(66 / 93)$ \\
\hline GM: galactomannan, BG: (1, 3)- $\beta$-D-glucan, IPA: invasive pulmonary \\
aspergillosis, BG/GM: IPA was diagnosed when one of both seromarkers \\
was positive in any one patient, BG+GM: IPA was diagnosed only when \\
both seromarkers were positive in any one patient, PPV: positive predictive \\
value, NPV: negative predictive value
\end{tabular}

both assays were positive for 10 patients.

Among the 11 of the 27 patients with probable or proven IPA with positive GM assays, 13 had positive results on the BG test, 19 patients had at least one positive results, and only five patients had positive results on both tests. Eight cases that were positive on the BG test were negative on the GM test, and six cases that were positive on the GM assay were negative on the $\mathrm{BG}$ assay.

The GM and BG median values for the survival and non- survival groups were not statistically different $(0.41$ vs. 0.92 , $\mathrm{p}=0.643$ and $71.8 \mathrm{pg} / \mathrm{mL}$ vs. $72.5 \mathrm{pg} / \mathrm{mL}, \mathrm{p}=0.173$ ).

\section{Discussion}

The GM antigen is an aspergillus-specific antigen that is released during the growth phase of invasive aspergillosis (IA). It has been considered as a useful surrogate marker for the diagnosis of IA, particularly in those patients with hematological malignancies or who have undergone hematopoietic stem cell transplantation (9). However, a recent retrospective study showed that the sensitivity of the GM assay was only $23.1 \%$, and the specificity was $76.1 \%$ when using a cut-off of 0.5 (12). Two other studies focusing on the value of GM antigen for the diagnosis of IPA in nonneutropenic patients reported that the GM assay had a relatively high sensitivity of $50.0-57.9 \%$ and a specificity of $84.2-87.3 \%$ when using a cut-off of $0.5(6,13)$. In our study, which included more proven IPA patients, a similar result was obtained with a sensitivity of $40.7 \%$ and a specificity of $89.4 \%$ when using the cut-off of 0.5 . This suggest that the GM assay might be less useful for the diagnosis of IA in non-hematological patients. 
Table 4. Prognostic Value of GM and BG Assays for IPA

\begin{tabular}{lccc}
\hline & Survival group $(\mathrm{n}=18)$ & Non-survival group $(\mathrm{n}=9)$ & $\mathrm{p}$ value \\
\hline GM [median (range)] & $0.41(0.18-3.29)$ & $0.92(0.21-1.82)$ & 0.643 \\
BG [median (range)] & $71.8(10.0-657.1)$ & $72.5(23.1-976.3)$ & 0.173 \\
\hline GM: galactomannan, BG: $(1,3)-\beta$-D-glucan, IPA: invasive pulmonary aspergillosis
\end{tabular}

The other important seromarker used for the early diagnosis is BG, which is a cell wall polysaccharide component specific for fungi except for zygomycetes and cryptococci (14). For hematological malignancies patients with IPA, the sensitivity and specificity of BG assay were $66 \%$ and $75.6 \%$, respectively, with a cut-off of $80 \mathrm{pg} / \mathrm{mL}(15)$. However, there are few existing studies on the value of the BG assay for non-neutropenic patients with IPA. In our study, the sensitivity and specificity of the BG assay were $48.1 \%$ and $78.8 \%$, respectively. Compared with neutropenic patients, the sensitivity for non-neutropenic patients was obviously lower.

One study reported that when the GM and BG assays were both positive in adult neutropenic patients, the specificity of each individual test could be improved without affecting the sensitivity for the diagnosis of IA (16). This was in contrast to our data, which demonstrated that positive results of both seromarkers had the highest specificity of $92.4 \%$ but a low sensitivity of $18.5 \%$. A possible reason for this might be that the serum samples were collected prematurely, as BG tended to become positive earlier than GM (16). In addition, Hachem et al. reported that due to the high sensitivity of the BG assay and the high specificity of the GM assay, the combination of both tests might be the best approach for diagnosing IA in hematologic malignancy patients (17). Our investigation indicated that the strategy of at least one positive result for the two tests in the diagnosis of IPA in non-neutropenic patients might have a relatively high sensitivity of $70.4 \%$, with a comparable specificity of $75.8 \%$. These findings were consistent with the results of other studies, such as GM combined with the clinical isolation of Aspergillus spp. or consecutive GM assays $(13,18)$. Considering that sputum cultures and consecutive GM tests would consume more time and increase cost, GM combined with BG might be more useful and efficient for early diagnosis.

Among patients with COPD, those who take corticosteroids are at high risk for IPA (4). Indeed, the most frequent underlying disease of IPA patients in the present study was COPD (18.5\%), and all of these patients received corticosteroid treatment. COPD was followed by solid organ malignancy and diabetes mellitus (both 11.1\%). In addition, $22.2 \%$ of the patients with IPA in our study had no basic diseases, which was a similar proportion to that of a previous study in non-neutropenic patients without any underlying diseases (19.2\%) (5). This finding should raise great concern and it therefore deserves sufficient attention.

There are several limitations associated with our study. Firstly, our patients were chosen from a single center, and the sample size was small. Secondly, the PPV might have been influenced by the prevalence of IPA, as the research had certain selectivity for a patient group with a high prevalence of IPA. Thirdly, our sampling strategy might have impacted the sensitivity of the GM and BG assays.

In conclusion, compared with the neutropenic patients, the serum GM and BG assays were less useful for the diagnosis of IPA in non-neutropenic patients. At least one positive result from the two tests appeared to be useful in diagnosing this patient group, but the value of this approach needs to be confirmed in future trials.

\section{The authors state that they have no Conflict of Interest (COI).}

\section{Acknowledgement}

The study was supported in part by Pfizer Inc. The sponsor had no role in the study design, conduct, analysis, or interpretation of results.

\section{Ethical Considerations}

This study was approved by the ethics committee of Chinese PLA General Hospital. Informed consent was obtained from all study subjects or their next of kin.

\section{References}

1. DePauw B, Walsh TJ, Donnelly JP, et al. Revised definitions of invasive fungal disease from the European Organization for Research and Treatment of Cancer/Invasive Fungal Infections Cooperative Group and the National Institute of Allergy and Infectious Diseases Mycoses Study Group (EORTC/MSG) Consensus Group. Clin Infect Dis 46: 1813-1821, 2008.

2. Smith NL, Denning DW. Underlying conditions in chronic pulmonary aspergillosis, including simple aspergilloma. Eur Respir J 37: 865-872, 2011.

3. Ader F, Nseir S, Le Berre R, et al. Invasive pulmonary aspergillosis in chronic obstructive pulmonary disease: an emerging fungal pathogen. Clin Microbiol Infect 11: 427-429, 2005.

4. Guinea J, Torres-Narbona M, Gijón P, et al. Pulmonary aspergillosis in patients with chronic obstructive pulmonary disease: Incidence, risk factors, and outcome. Clin Microbiol Infect 16: 870877, 2010.

5. Dai Z, Zhao H, Cai S. Invasive pulmonary aspergillosis in nonneutropenic patients with and without underlying disease: a singlecentre retrospective analysis of 52 subjects. Respirology 18: 323331, 2013.

6. Xu PF, Zhou JY, Zhou H, Shen P. Serum antigens assay combined with chest CT scan in diagnosis of invasive pulmonary aspergillosis. Zhejiang Da Xue Xue Bao Yi Xue Ban [Journal of Zhejiang University (Medical Sciences)] 41: 332-338, 2012 (in Chinese, Abstract in English).

7. Karageorgopoulos DE, Vouloumanou EK, Ntziora F, Michalopou$\operatorname{los}$ A, Rafailidis PI, Falagas ME. $\beta$-D-glucan assay for the diagnosis of invasive fungal infections: a meta-analysis. Clin Infect Dis 
52: 750-770, 2011.

8. Lu Y, Chen YQ, Guo YL, Qin SM, Wu C, Wang K. Diagnosis of invasive fungal disease using serum $(1 \rightarrow 3)-\beta$-D-glucan: a bivariate meta-analysis. Intern Med 50: 2783-2791, 2011.

9. Pfeiffer CD, Fine JP, Safdar N. Diagnosis of invasive aspergillosis using a galactomannan assay: a meta-analysis. Clin Infect Dis $\mathbf{4 2}$ : 1417-1427, 2006.

10. Pickering JW, Sant HW, Bowles CA, Roberts WL, Woods GL. Evaluation of a (1->3)-beta-D-glucan assay for diagnosis of invasive fungal infections. J Clin Microbiol 43: 5957-5962, 2005.

11. Lai CC, Hsu HL, Lee LN, Hsueh PR. Assessment of Platelia Aspergillus enzyme immunoassay for the diagnosis of invasive aspergillosis. J Microbiol Immunol Infect 40: 148-153, 2007.

12. Ku NS, Han SH, Choi JY, et al. Diagnostic value of the serum galactomannan assay for invasive aspergillosis: It is less useful in non-haematological patients. Scand J Infect Dis 44: 600-604, 2012.

13. He H, Ding L, Chang S, Li F, Zhan Q. Value of consecutive galactomannan determinations for the diagnosis and prognosis of invasive pulmonary aspergillosis in critically ill chronic obstructive pulmonary disease. Med Mycol 49: 345-351, 2011.

14. Persat F, Ranque S, Derouin F, Michel-Nguyen A, Picot S, Sula- hian A. Contribution of the (1-->3)-beta-D-glucan assay for diagnosis of invasive fungal infections. J Clin Microbiol 46: 10091013, 2008.

15. Metan G, Koç AN, Atalay A. What should be the optimal cut-off of serum 1,3- $\beta$-D-glucan for the detection of invasive pulmonary aspergillosisin patients with haematological malignancies? Scand J Infect Dis 44: 330-336, 2012.

16. Pazos C, Ponton J, Del Palacio A. Contribution of (1-3)- $\beta-D-$ glucan chromogenic assay to diagnosis and therapeutic monitoring of invasive aspergillosis in neutropenic adult patients: a comparison with serial screening for circulating galactomannan. J Clin Microb 43: 299-305, 2005.

17. Hachem RY, Kontoyiannis DP, Chemaly RF, Jiang Y, Reitzel R, Raad I. Utility of galactomannan enzyme immunoassay and $(1,3)$ beta-D-glucan in diagnosis of invasive fungal infections: low sensitivity for Aspergillus fumigatus infection in hematologic malignancy patients. J Clin Microbiol 47: 129-133, 2009.

18. Guinea J, Jensen J, Peláez T, et al. Value of a single galactomannan determination (Platelia) for the diagnosis of invasive aspergillosis in non-hematological patients with clinical isolation of Aspergillus spp. Med Mycol 46: 575-579, 2008.

(C) 2014 The Japanese Society of Internal Medicine http://www.naika.or.jp/imonline/index.html 